\title{
EXPERIMENTS ON WHISTLER WAVE FILAMENTATION AND VLF HISS IN A LABORATORY PLASMA (*)
}

\author{
R. L. STENZEL (**) \\ TRW Systems, Redondo Beach, CA 90278, U. S. A.
}

\begin{abstract}
Résumé. - Avec le développement de grande source à plasma magnétisé, il est devenu possible d'étudier des problèmes de physique des plasmas spatiaux en laboratoire. D'abord ont été explorés les effets non linéaires associés à l'excitation de modes siffleurs de grande amplitude. On trouve que la pression de rayonnement de l'onde et les effets thermiques produisent une dépression de densité alignée avec le champ, dans laquelle l'onde devient complètement piégée. Des filaments hyperfins sont observés, dont les diamètres sont petits par rapport à la longueur d'onde parallèle. Ensuite, a été étudiée la stabilité des modes siffleurs obliques en présence d'un faisceau d'électrons. Une instabilité de siffleur, de large bande, est observée et identifiée comme une interaction Cerenkov entre les électrons du faisceau et les siffleurs se propageant auprès du cône de résonance. Ces observations confirment le présent modèle pour la production des signaux de très basse fréquence apparaissant à l’aube.
\end{abstract}

\begin{abstract}
With the development of a large magnetized plasma source it has become possible to investigate space plasma physics problems in the laboratory. First, the nonlinear effects associated with the excitation of a large amplitude whistler wave have been explored. It is found that the radiation pressure of the wave and thermal effects give rise to a field-aligned density depression in which the wave becomes completely trapped. Hyperfine filaments with diameters small compared with the parallel wavelength are observed. Second, the stability of oblique whistler waves in the presence of an electron beam has been studied. A broadband whistler instability is observed and identified as a Cherenkov interaction between beam electrons and whistlers propagating near the resonance cone. These observations confirm the present model for the generation of VLF hiss in the aurora.
\end{abstract}

1. Introduction. - In order to investigate space plasma physics problems such as whistler waves in the laboratory a fundamental requirement is a plasma source of dimensions large compared with the characteristic damping or growth length of the wave. In the present work a quiescent uniform plasma column of approximately $1 / 2 \mathrm{~m}$ diameter and $3 \mathrm{~m}$ length has been constructed, corresponding to approximately 10 electromagnetic whistler wavelengths across and 60 wavelengths along the magnetic field, respectively. The plasma can be penetrated by an electron beam of the same diameter. These source dimensions are adequate for wave propagation experiments free from boundary effects.

The exictation and propagation of a large amplitude whistler wave is a problem of fundamental and practical interest. Although the stability of electromagnetic whistlers has been analyzed in theory and computer

(*) This work was supported in part by the National Aeronautics and Space Administration under Contract Nos. NASA31175, NASW-2953, and in part by TRW Independent Research and Development Funds.

(**) Department of Physics, University of California, Los Angeles, California 90024, U. S. A. simulations [1-3], it is difficult to predict the nonlinear effects for a spectrum of oblique whistlers excited by a finite-size antenna. Is the present experiment the phase and amplitude distribution of antenna-launched whistlers is investigated at both small and large wave amplitudes. At small amplitudes the ducting of whistlers [4] in field-aligned density non-uniformities is demonstrated. At large amplitudes the wave modifies by its radiation pressure the initially uniform plasma so as to create a field-aligned density trough. The wave refracts into this path which further enhances the nonlinear process. A filamentation instability [5-8] results whereby the initially diverging radiation pattern collapses into a narrow channel of high wave intensity. Pencil-shaped antenna radiation patterns are observed. The wave-generated duct is also used to guide small amplitude whistlers over a wide frequency range and ordinary electromagnetic waves near the average plasma frequency of the duct

$$
\left(\omega_{\text {pmin }}<\omega<\omega_{\text {pmax }}\right) \text {. }
$$

Since whistlers propagate, in general, oblique to the magnetic field the stability of these modes is an important topic. While transverse electromagnetic 
whistlers are unstable to perpendicular temperature anisotropies oblique whistlers can be subject to electrostatic instabilities due to an electron beam $[9,10]$. A Cherenkov-type instability [11] arises when the beam velocity matches the parallel phase velocity component of an oblique whistler. For slow electron beams $\left(v_{\mathrm{b}} \ll c \omega_{\mathrm{c}} / \omega_{\mathrm{p}}\right)$ the whistler wave propagates near the oblique resonance $\left(\cos \theta \simeq \omega / \omega_{c}\right)$. Since the waveparticle resonance condition can be satisfied at different angles for any frequency between the lower hybrid and the electron cyclotron resonance the instability has a broadband character.

Experimentally, the instability is produced by injecting an energetic electron beam into a quiescent afterglow plasma [12]. The emission of broadband rf noise upon beam injection is observed. By analyzing the frequency and wave vector spectrum, i. e. the dispersion $\omega(\mathbf{k})$, the noise is identified as whistlers propagating near the oblique resonance with parallel phase velocity matched by the beam velocity, as predicted by theory. Coherent test waves are launched from a small antenna in the unstable plasma. In the direction opposing the beam the point source excites a familiar resonance cone [13, 14] but with the beam a single oblique whistler mode is excited, i. e. the most unstable mode, which grows oblique to the beam at an angle $\theta_{c} \lesssim \operatorname{arc} \sin \left(\omega / \omega_{c}\right)$, propagates at nearly the complementary angle and is in resonance with the beam $\left(\omega / k_{\|} \lesssim v_{\mathrm{b}}\right)$.

First, in section 2, the experimental arrangement is described. Then, in section 3 , the observations on linear and nonlinear whistler wave propagation in a quiescent plasma are presented and discussed. In section 4 the results for the instability of oblique whistler waves are presented and the connections with auroral hiss [15] are shown. Finally, section 5 presents a conclusion on these space related laboratory experiments [16].

2. Experimental setup and measurement techniques. - A schematic view of the plasma device is shown in figure 1 . The vacuum chamber of $1 \mathrm{~m}$ diameter and $3.5 \mathrm{~m}$ length contains a magnetized plasma column uniform to within $\pm 10 \%$ over $45 \mathrm{~cm}$ in diameter and $300 \mathrm{~cm}$ in length. An unusually large $(50 \mathrm{~cm}$

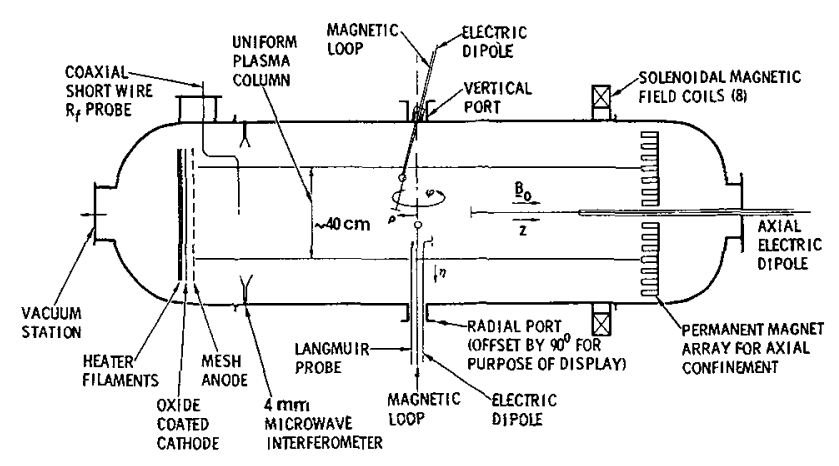

FIG. 1. - Schematic view of the plasma device. diameter), indirectly heated ( $9 \mathrm{~kW}$ heater power), oxide coated $(\mathrm{BaO}, \mathrm{SrO})$ cathode provides abundant (up to $1 \mathrm{~A} / \mathrm{cm}^{2}$ ) energetic $(\sim 50 \mathrm{eV})$, collisionless (mean free path $>3 \mathrm{~m}$ ) electrons which produce a uniform plasma by impact ionization with background neutrals (Krypton, $\sim 2 \times 10^{-4}$ torr). The plasma is confined by a uniform $\left(\delta B / B_{0}<0.5 \%\right)$ axial magnetic field $\left(0<B_{0}<150 \mathrm{G}\right)$ produced by a set of 8 external solenoidal coils driven with a transistor-regulated power supply $(15 \mathrm{~V}, 2000 \mathrm{~A}$; temporal stability $\delta B / B_{0}<10^{-4}$ ). Axial plasma confinement is achieved with a multimirror permanent magnet wall consisting of 200 samarium cobalt magnets $\left(B_{\max } \simeq 4 \mathrm{kG}\right)$ mounted in arrays of opposite polarity. Due to the magnetic field confinement and the relatively efficient plasma production a large volume (500 liter), high density $\left(n_{\mathrm{c}} \simeq 10^{12} \mathrm{~cm}^{-3}\right.$ ), collisionless $\left(v_{\mathrm{en}} / \omega_{\mathrm{c}} \simeq 5 \times 10^{-3}\right)$ plasma is produced at moderate discharge powers $(40 \mathrm{~V} \times 250 \mathrm{~A}=10 \mathrm{~kW})$. Density and magnetic field are independently variable, the electron temperature varies from $k T_{\mathrm{e}} \simeq 2 \mathrm{eV} \simeq 10 k T_{\mathrm{i}}$ during the discharge to $k T_{\mathrm{e}} \simeq 0.2 \mathrm{eV} \simeq k T_{\mathrm{i}}$ in the late afterglow of a pulsed discharge. Pulsed operation (2 $\mathrm{kJ}$ capacitor bank, $500 \mathrm{~A}$ transistor switch) is preferred since it optimizes the lifetime of the cathode ( $\sim 2$ months) and yields a quiescent Maxwellian afterglow plasma highly suited for wave experiments. The $e$-folding decay time constants for density and temperature are $\tau_{\mathrm{n}} \simeq 10 \mathrm{~ms}, \tau_{\mathrm{E}} \simeq 1 \mathrm{~ms}$, respectively. The primary electrons are lost within the first $50 \mu \mathrm{s}$ after the end of the discharge pulse.

The plasma diagnostics consists of a $70 \mathrm{GHz}$ $(4 \mathrm{~mm})$ microwave interferometer for radially averaged time resolved density measurements, of various Langmuir probes for time and space resolved density, temperature and distribution function measurements, and of a microwave resonator probe [17] for local density measurements.

Whistler waves are excited and detected with various antennas inserted into the center of the plasma column through axial and orthogonal radial ports. The antennas are balanced electric dipoles (length $l=1.5 \mathrm{~cm}$ and $5 \mathrm{~cm}$ ), shielded magnetic loops (diameter $1.6 \mathrm{~cm}$ ) and simple short wire coax-fed antennas $(5 \mathrm{~mm}$ exposed wire of $50 \Omega$ semi-rigid coax cable). The antennas are tested for their proper dipole response in simple field geometries (parallelplate capacitor for dipole, linear current conductor for loop) in air. The magnetic loop antenna is calibrated and absolute of $B$-field measurements are performed inside the plasma whose permeability does not affect the calibration. Electric fields have only been measured relatively. In single frequency operation the exciter antenna is matched to the rf amplifier by a double-stub tuner.

The experimental technique is schematically shown in figure 2. The plasma is pulsed repetitively $(5 \mathrm{~ms}$ on, $500 \mathrm{~ms}$ off) and the whistler waves are propagated in the quiescent afterglow plasma. All data are 

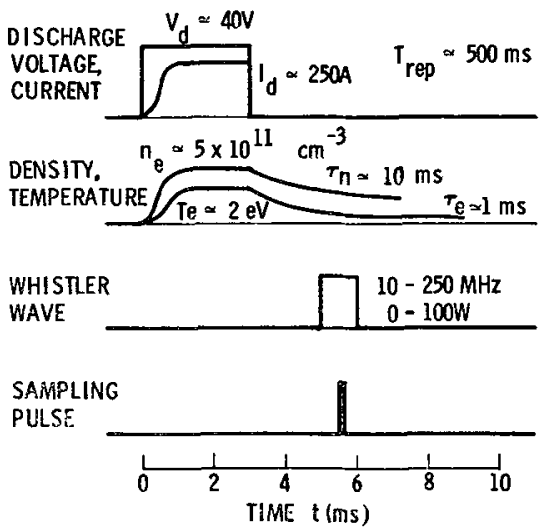

FIG. 2. - Typical time sequence of the pulsed plasma experiment.

sampled with a boxcar integrator and averaged over many highly reproducible afterglow periods. The slow density and temperature decay is negligible compared to the time scale of the experiment.

In the experiment on whistler instabilities the beam is produced by applying a second independently controlled pulse to the cathode so as to inject electrons into an initially quiescent afterglow plasma.

Interferometer techniques are used to obtain the spatial amplitude and phase distribution

$$
\left[\propto A_{(z)} \cos (k z)\right]
$$

of monochromatic waves. For amplitude measurements without phase factor a spectrum analyzer tuned manually to the incident frequency is used. For the analysis fo the beam generated rf emission two identical coaxial probes movable along and across $\mathbf{B}_{0}$ are used. With narrowband amplifiers, delay line and multiplier the cross spectral function is obtained which yields the dispersion $\omega(\mathbf{k})$.

3. Whistler wave filamentation. - Before presenting the observations on self-focusing it is appropriate to show the process of whistler wave ducting by field-aligned density non-uniformities in the linear regime.

3.1 Linear Wave Ducting. - Figure 3 displays raw data interferometer traces, i. e. amplitude and phase, $B_{1}(z) \cos \left(k_{\|} z\right)$, versus axial position $z$ for a small amplitude whistler wave. In figure $3 a$, the wave is launched in a highly uniform plasma. The axial wavelength $\left(\lambda_{\|} \simeq 4 \mathrm{~cm}\right)$ is constant indicating that density and magnetic field do not vary in axial direction. The amplitude decays strongly with distance from the exciter (magnetic loop antenna). This decay is not due to collisional or cyclotron damping but due to the geometric divergence of the energy flux. With a finite size antenna (loop diameter $d \simeq \lambda_{\mid /} / 2$ ) one cannot launch a plane wave. However, as shown in figure $3 b$, one can excite a non diverging whistler

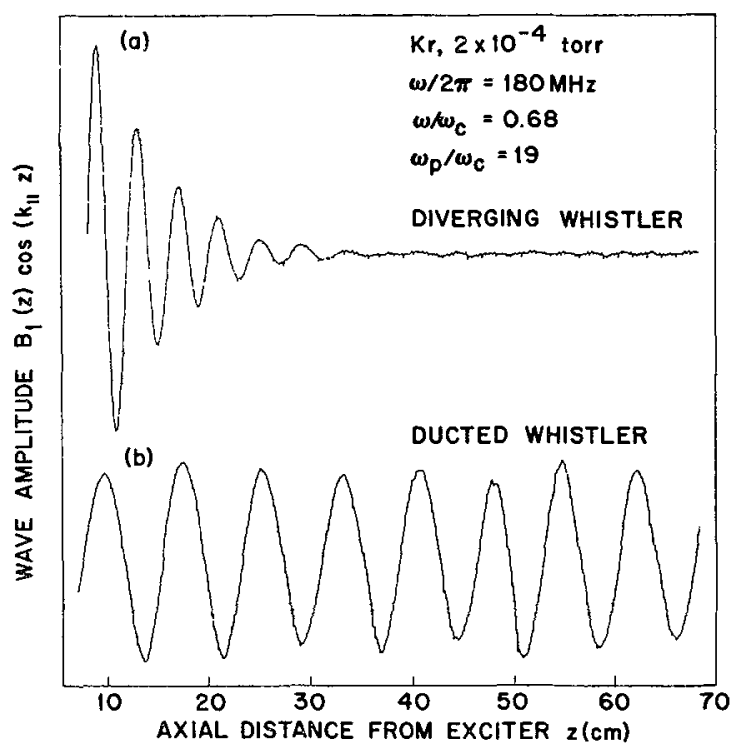

FIG. 3. - Raw data of axial whistler wave interferometer traces. In a uniform plasma (a) the amplitude decays due to the divergence of the energy flux ; in a density trough $(b)$ the wave is ducted and propagates at a constant amplitude in a collisionless plasma.

wave, i. e. a ducted whistler. The duct in this case corresponds to a field aligned density trough

$\left(\delta n_{\mathrm{e}} / n_{\mathrm{e}} \simeq-30 \% ; \quad\right.$ radial width $\left.\quad \Delta r \simeq 5 \mathrm{~cm}<\lambda_{\|}\right)$

produced by launching a high power whistler wave from the same antenna, as will be shown in detail below. In the absence of geometric spread there is virtually no damping observable which indicates that the plasma is not only collisionless but also free from low-frequency density fluctuations which can cause damping by random phase modulation. Moreover, since the data are averaged over many afterglow periods the plasma stability and reproducibility are excellent.

The ducting process arises from wave refraction by radial density gradients [18]. By measuring the amplitude and phase distribution of a whistler wave launched on a density gradient the refraction process can be experimentally verified. The results are summarized in figures $4 a-c$. For reference, the radial density profile is shown on the right. The density nonuniformity is intentionally produced by nonuniform cathode emission. Figure $4 a$ displays the phase contours, i. e. maxima and minima of the interferometer traces in a plane along and across $\mathbf{B}_{0}$. The magnetic loop antenna shown to scale by the circle at the origin $(\Delta r=\Delta z=0)$ is located in the middle of the radial density gradient with the objective of studying crest or trough ducting. First, one notices that in contrast to free space, the wave fronts are concave with respect to the exciter, i. e. the wave vectors are converging away from the exciter. This apparent focusing effect is the result of the different directions of energy flow and wave propagation in 

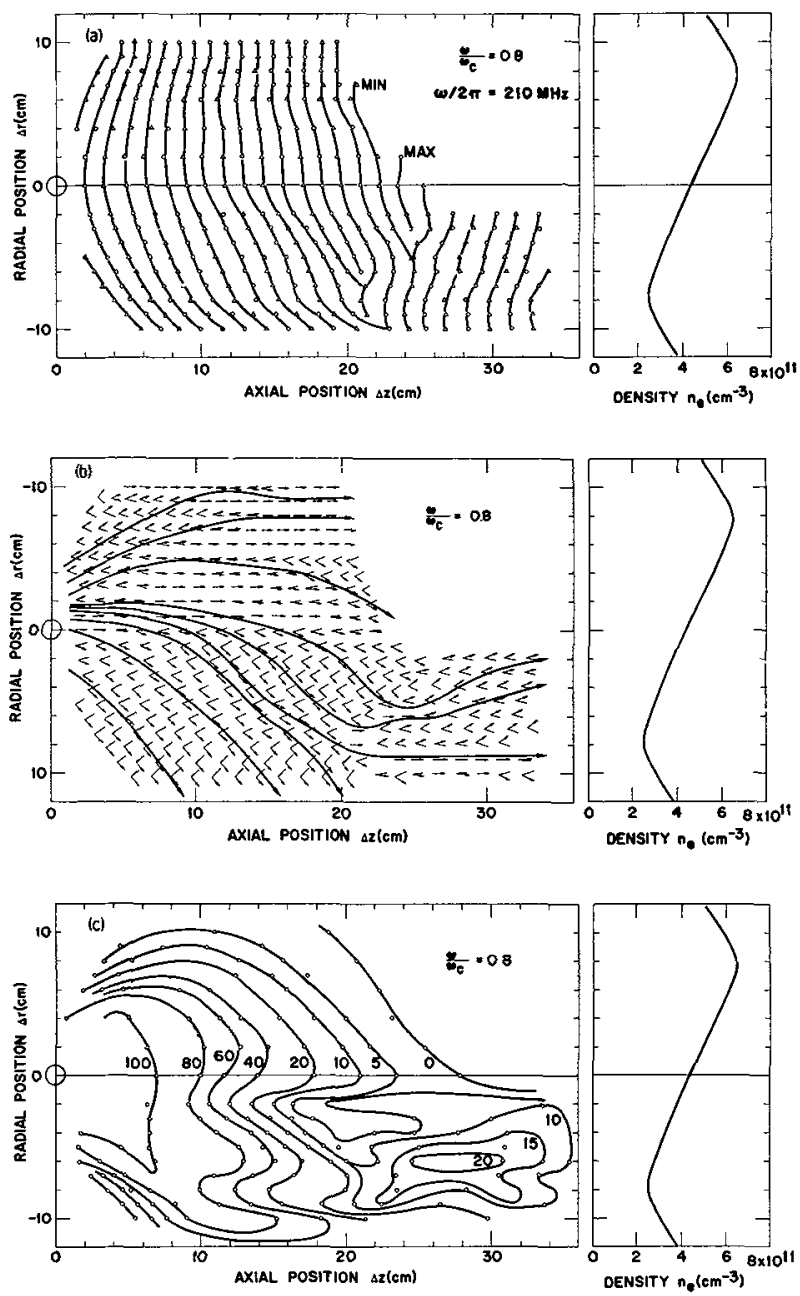

FIG. 4. - Radiation pattern of a magnetic loop antenna (indicated to scale by circle at origin) at $\omega / \omega_{c}>0.5$ in a non uniform plasma with radial density profile indicated on the right hand side. (a) Contours of constant phase (maxima and minima of axial interferometer traces) ; (b) Vector field of wave normals (small straight lines without arrows) constructed from the measured phase contours, and vector field of rays (small lines with arrows) calculated for each wave normal direction from equation (1). The heavy solid lines are a few characteristic rays. The ray tracing indicates the refraction of a whistler wave at $\omega / \omega_{\mathfrak{c}}>0.5$ into a density trough. (c) Contours of constant relative amplitudes. The asymmetric amplitude distribution is the result of whistler wave ducting in a density trough.

an anisotropic medium. For $0.5<\omega / \omega_{\mathrm{c}}<1$ the direction of the ray (group velocity, energy flow) and the wave normal (phase velocity) are on opposite sides with respect to the static magnetic field [4]. Since by causality the rays diverge from the exciter, the wave normals are converging. The focusing of the phases does, of course, not imply an amplitude enhancement.

After a few wavelengths from the antenna the phase fronts develop different shapes in the crest and trough region. The wave refracts and since the density gradient scale length is large compared with the wavelength $\left(n / \nabla_{\perp} n \simeq 8 \lambda_{\| l}\right)$ one can perform a ray tracing analysis. In figure $4 b$ a vector field of wave normals (thin straight lines without arrows) corresponding to the phase contours of figure $4 a$ has been constructed. Then, for each wave normal the ray direction has been determined from the expression [4]

$$
(\theta-\alpha)=\arctan \frac{\sin \theta\left(\cos \theta-2 \omega / \omega_{c}\right)}{1+\cos \theta\left(\cos \theta-2 \omega / \omega_{c}\right)}
$$

where $\theta$ is the wave normal angle and $(\theta-\alpha)$ the ray angle with respect to $\mathbf{B}_{\mathbf{0}}$. The vector field of rays (thin straight lines with arrows) makes ray tracing possible and a few characteristic rays (thick solid lines with arrows) have been indicated. There is a general tendency of the initially divergent bundle of rays to refract toward the trough. Some rays (middle three) enter the trough and begin to perform snake-like motions, other rays (bottom three) which have a steep angle of incidence miss the trough and become evanescent modes. This wave refraction process leads to an amplitude distribution which is shown in figure $4 c$. The observed amplitude contours are highly asymmetric bending deep into the trough region. The wave amplitude is always larger in the low density region, and beyond about 10 wavelengths the wave has completely refracted into the trough. This observation is a direct verification of the generally accepted theoretical picture of whistler wave ducting.

Although the ducting process based upon wave refraction is only applicable to density troughs large compared with the wavelength small diameter ducts also exhibit ducting properties as shown by an eigenmode analysis [19].

3.2 Nonlinear Ducting. - When axial interferometer traces of large amplitude whistlers are taken the apparent wave damping is observed to decrease with increasing wave amplitude. Simultaneously, the amplitude distribution across $\mathbf{B}_{0}$ narrows indicating that the decrease in the divergence of the Poynting flux causes the slow axial amplitude decay. Finally, intense whistlers are found to propagate without divergence at a constant amplitude along $\mathbf{B}_{0}$. Figure 5 shows a comparison of the wave intensity vs. radial position at different axial distances from the exciter for a linear (Fig. 5a and nonlinear whistler wave Fig. $5 b$ ). While the small amplitude wave has a diverging energy flow the large amplitude wave is confined to a narrow region across $\mathbf{B}_{0}$ which does not broaden with increasing distance from the exciter antenna. Thus the large amplitude wave has created a duct or filament in which the energy is confined and the wave amplitude remains constant in the absence of collisions. For intermediate power levels the wave divergence lies between the two cases shown.

The temporal evolution of the duct formation is shown in figure 6 which displays axial interferometer traces of an intense whistler sampled at different times $t_{\mathrm{s}}$ after the step-like turn-on of the wave burst. The filamentation process is found to occur on a time scale slow compared with the wave transit time 


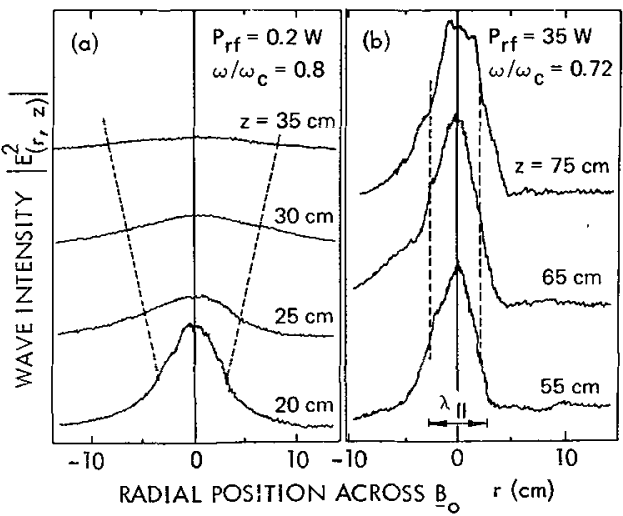

Fig. 5. - Wave intensity across the magnetic field at different axial distance from the dipole exciter antenna. In the linear regime (Fig. 5a) the wave intensity decreases rapidly with distance $z$ due to the diverging energy flow. In the nonlinear regime (Fig. $5 b$ ) the wave intensity is confined to a narrow, field-aligned nondiverging filament.

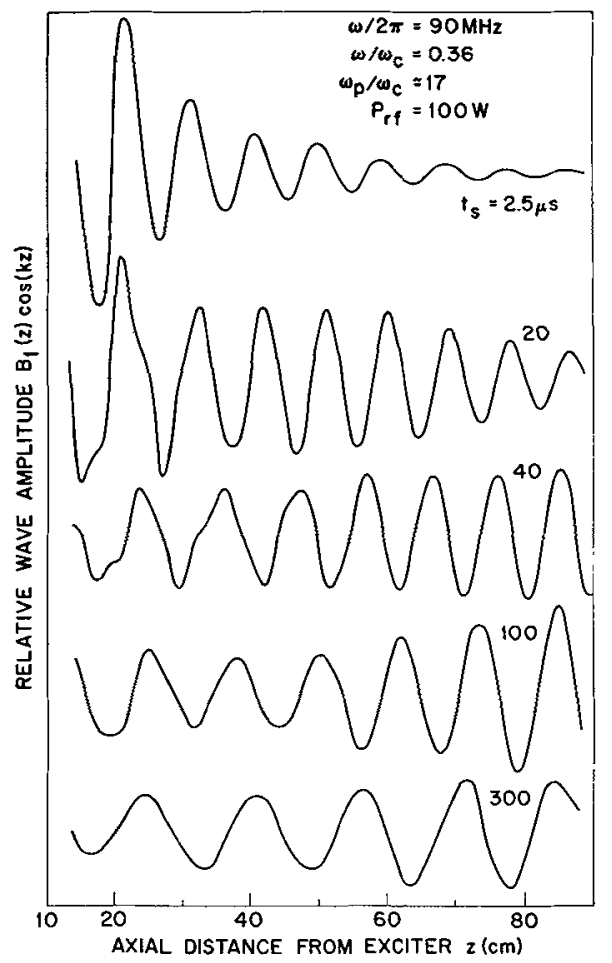

FIG. 6. - Axial interferometer traces of a high power whistler wave sampled at different times $t_{\mathrm{s}}$ after the step-like turn-on of the wave. The wave self-focuses within $40 \mu \mathrm{s}$.

through the device. At early time (top trace, $\left.t_{\mathrm{s}}=2.5 \mu \mathrm{s}\right)$ the amplitude decay is the same as in the linear case. With increasing time the amplitude decay becomes smaller, vanishes and reverses into a small growth.

The amplitude and phase distribution across the duct at a distance $z=70 \mathrm{~cm}$ from the antenna is shown in figure 7. The $3 \mathrm{~dB}$ width of the duct is $\Delta r \simeq 2 \mathrm{~cm}$ which is considerably smaller than the axial wavelength $\left(\lambda_{\|} \simeq 10 \mathrm{~cm}\right)$. The phase fronts are essentially plane and normal to $\mathbf{B}_{0}$. Figure 8

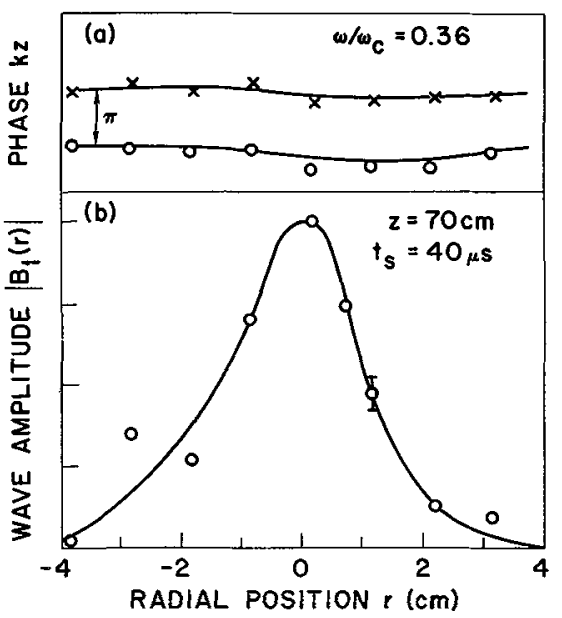

FIG. 7. - Phase (a) and amplitude (b) distribution across the magnetic field of the self-focused whistler wave. Parameters as in figure 6.

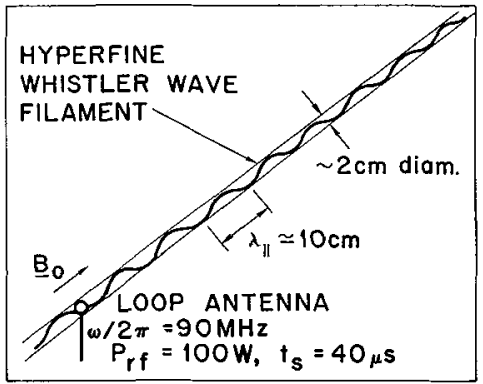

Frg. 8. - Schematic view of the radiation pattern of an intense whistler as derived from the data of figures 6 and 7. The selffocused whistler propagates within a slightly constricting filament of diameter small compared with the axial wavelength.

summarizes the amplitude measurements at $t_{\mathrm{s}}=40 \mu \mathrm{s}$ and displays schematically the resultant radiation pattern. The striking result is that the wave is confined to a hyperfine filament with characteristic wave numbers $k_{\perp} \gg k_{\|} \gg k_{0}$ where $k_{0}=\omega / c$. The duct slightly constricts with increasing distance from the antenna which causes the small axial amplitude growth, characteristic for a self-focusing process. The fact that wave propagation without cutoff is possible in such a narrow duct is related to the wave dispersion. Unlike electromagnetic waves oblique whistlers have a wavenumber which increases with propagation angle and exhibits a resonance $(k \rightarrow \infty)$ at $\theta \simeq \arccos \left(\omega / \omega_{\mathrm{c}}\right)$. Although the observed phase fronts in the duct are normal to $\mathbf{B}_{0}$ the wave can be considered as a superposition of highly oblique whistlers.

The slow duct formation indicates that the zeroorder plasma parameters are changed by the wave. Time and space resolved density measurements show that the duct corresponds to a field aligned density depression. Figure $9 a$ displays the radial density profile sampled at different times $t_{\mathrm{s}}$ after the turn-on of a large amplitude whistler wave. The initial profile 

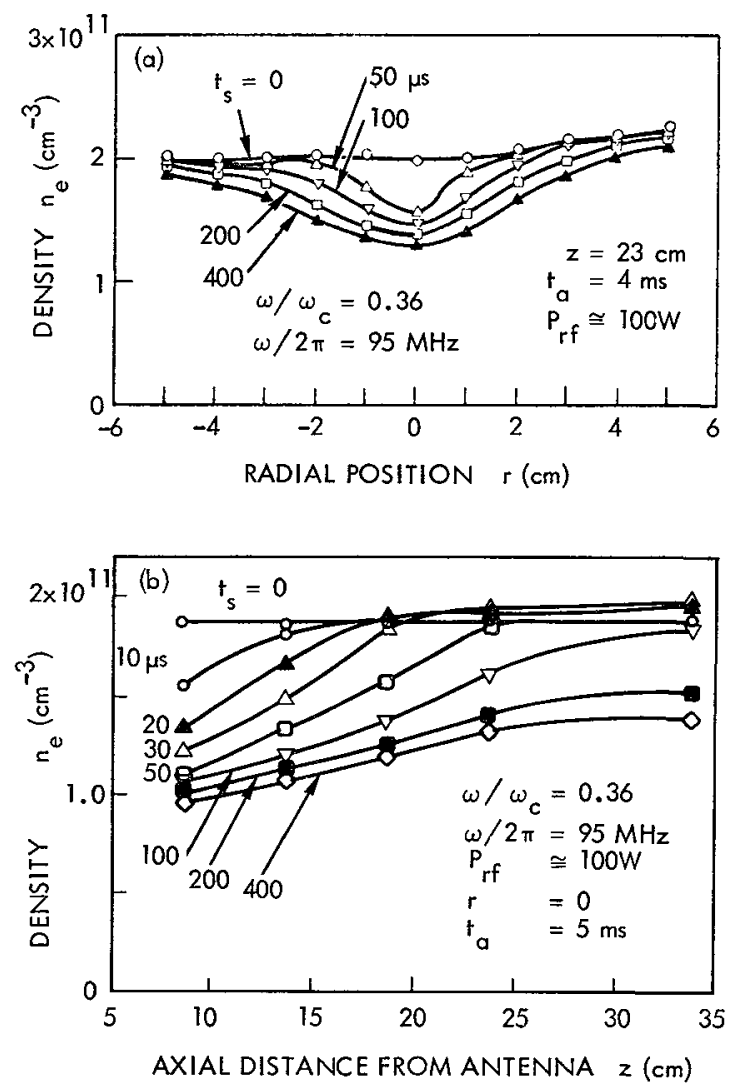

FiG. 9. - Density profiles across (Fig. 9a) and along (Fig. 9b) the magnetic field sampled at different times $t_{\mathrm{s}}$ after the turnon of the large amplitude wave applied to a $1.6 \mathrm{~cm}$ dia. magnetic loop antenna at $r=0, z=0$. The density is measured with a microwave resonator probe [17] which is insensitive to the electron temperature profile.

is flat, but in time a density depression develops which deepens and widens. The density minimum corresponds to the location of the antenna, the half-width is wider but on the order of the antenna dimensions across $\mathbf{B}_{0}$. The axial profile (Fig. $9 b$ ) shows that the density depression originates from the antenna and subsequently spreads out along $\mathbf{B}_{0}$ to form eventually a long field aligned density trough. After the turn-off of the whistler wave burst the density perturbation relaxes on a slow time scale determined by ion diffusion.

Not only the electron density but also the electron temperature is modified. In the duct the electrons are heated $\left[\Delta T_{\mathrm{e}} / T_{\mathrm{e}}=0(1)\right]$, in particular, by whistlers near cyclotron resonance. The heated electrons exhibit a small anisotropy $\left(T_{\mathrm{e} \perp} / T_{\mathrm{e} \|} \simeq 1.4\right)$. Heating occurs only during the duct formation $(t \lesssim 100 \mu \mathrm{s})$ and the temperature remains constant while the large amplitude wave propagates undamped through the duct. The axial temperature gradients are, in general, much smaller than the axial density gradients indicating that the density depression is not simply the result of the electron thermal pressure but an axial force such as the wave radiation pressure. After the turn-off of the rf pulse, the plasma parameters in the duct relax assuming almost the unperturbed values in the absence of the rf pulse. This behavior indicates that the plasma displaced from the duct has not been lost but only redistributed. The afterglow plasma is source free and almost sink free on the time scale of the experiment $\left(t_{\exp } \sim 1 \mathrm{~ms}\right.$, density decay time $t_{\mathrm{n}} \simeq 10 \mathrm{~ms}$ ).

The frequency dependence of the observed nonlinear effects is relatively weak. Duct formation is observed both above and below $\omega / \omega_{\mathrm{c}}=0.5$ but at low frequencies $\left(\omega / \omega_{\mathrm{c}}<0.2\right)$ the ducting becomes gradually weaker. This may be related to the small size of the antenna or duct width compared with the whistler wavelength and to the change of the wave dispersion properties. At low frequencies whistler waves duct along density crests rather than troughs.

Not only the high power whistler wave is effectively guided along the density trough but also small amplitude whistlers over a wide frequency range

$$
\left(0.25<\omega / \omega_{\mathrm{c}}<1\right) .
$$

These can be excited by the same antenna in the presence of the high power pulse. Figure $3 b$ gave an example for the ducting of a linear whistler wave $\left(\omega / \omega_{c}=0.68\right)$ in the trough produced by a nonlinear wave at a different frequency $\left(\omega / \omega_{c}=0.36\right)$. The field-aligned density depression also forms a perfect waveguide for high frequency $\left(\omega \simeq \omega_{\mathrm{p}}\right)$ electromagnetic wave which are cut off in the uniform plasma but are above cut off in the duct. Figure $10 a$ shows schematically how a duct can be used as a plasma waveguide. Figure $10 b$ displays axial interferometer traces of the microwave signal at different times of the duct formation. While the microwave signal is initially evanescent it propagates finally at a constant amplitude along the duct of diameter

$$
d \gtrsim \lambda_{\|} / 2 \simeq 5 \mathrm{~cm} \text {. }
$$

Figure $10 c$ confirms that the wave intensity at a large distance from the source is well confined to the duct.

Finally, the whistler wave intensity has been determined both from the radiated power and from in-situ wave magnetic field measurements with a calibrated loop antenna. The radiation efficiency of the antennas is obtained by measuring incident and reflected voltages on the antenna transmission line. In order to discriminate against cable losses, sheath losses and near-zone power dissipation a differential measurement is employed. The change in the absorbed power is measured when the magnetic loop antenna is rotated from the optimum polarization $\left(\mathbf{B}_{1} \perp \mathbf{B}_{0}\right)$ to the polarization for minimum radiation $\left(\mathbf{B}_{1} \| \mathbf{B}_{0}\right)$. The increment in the absorbed power which is the actually radiated power amounts to approx. $10 \%$ of the applied power. Thus, for an applied power level of $P_{\mathrm{inc}}=100 \mathrm{~W}$ and a duct of diameter $d \simeq 2 \mathrm{~cm}$ 

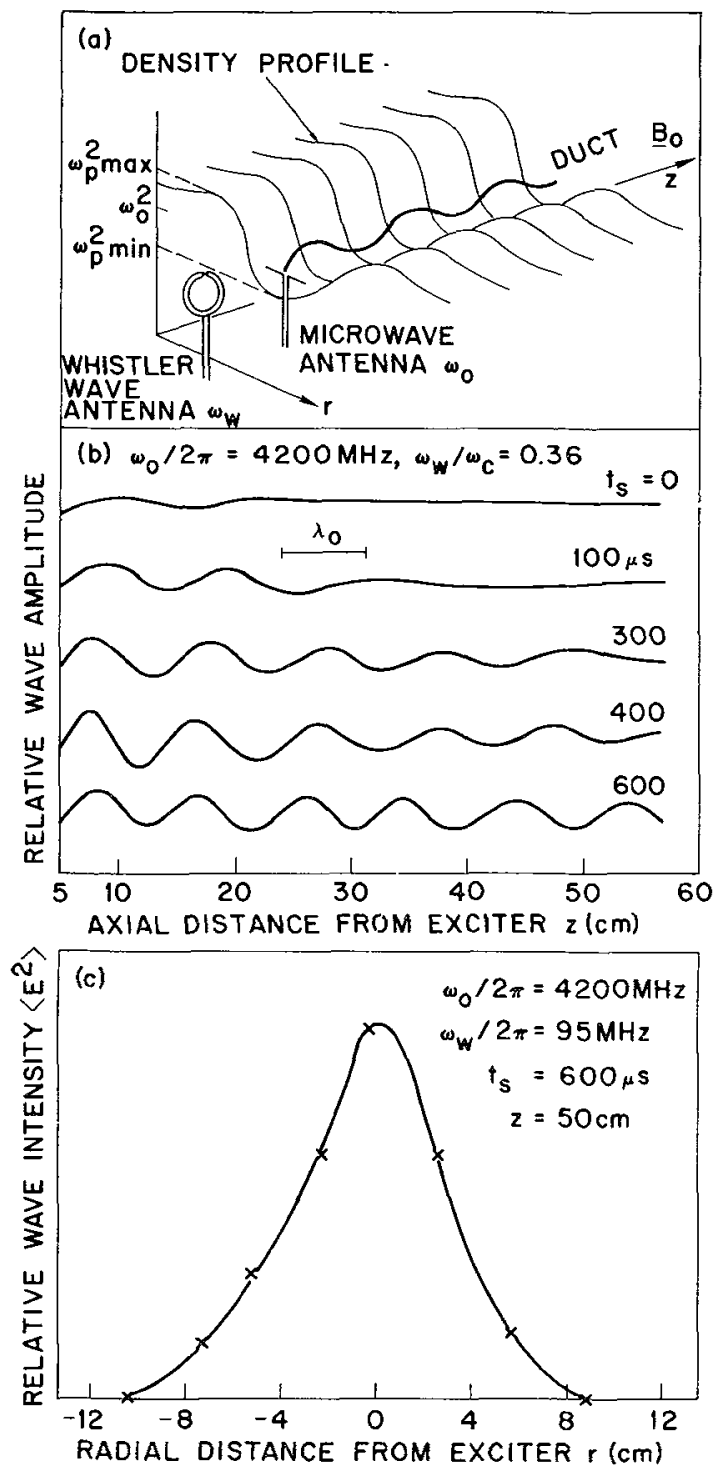

FIG. 10. - (a) Schematic arrangement how to use a whistler wave duct as a plasma waveguide for high frequency electromagnetic waves. (b) Axial interferometer traces of a microwave signal at different times $t_{\mathrm{s}}$ after turn-on of the intense whistler wave. As the duct is formed the initially evanescent test wave propagates at a constant amplitude. (c) The radial intensity profile at a large distance from the exciter shows that the microwave signal is confined to the duct.

the Poynting flux is $S=\left(P_{\mathrm{rad}} / 2\right) /\left(\pi d^{2} / 4\right) \simeq 1.6 \mathrm{~W} / \mathrm{cm}^{2}$, the wave energy density

$$
B_{1}^{2} / \mu_{0}=(n / c) S \simeq 2.7 \times 10^{-9} \mathrm{Joule} / \mathrm{cm}^{3}
$$

for a typical refractive index of $n=50$ and the wave magnetic field $B_{1} \simeq 0.5 \mathrm{G}$ in a plane wave approximation. The oscillating magnetic field in the loop center is still larger since the loop is at a current node with

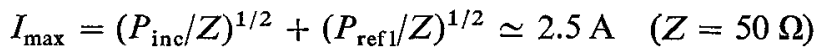

resulting in $B_{\max }=\mu_{0} I_{\max } /(2 a) \simeq 1 \mathrm{G}$ (loop diameter $2 a=1.6 \mathrm{~cm}$ ). For typical afterglow plasma parameters $\left(n_{\mathrm{e}} \simeq 2 \times 10^{11} \mathrm{~cm}^{-3}, \quad k T_{\mathrm{e}} \simeq 0.3 \mathrm{eV}, \quad B_{0} \simeq 90 \mathrm{G}\right)$ these estimates yield a wave pressure normalized to the particle pressure

$$
\left(B_{1}^{2} / \mu_{0}\right) /\left(n_{\mathrm{e}} k T_{\mathrm{e}}\right) \simeq 25 \%
$$

and a ratio of oscillating $B_{1}$-field to static $B_{0}$-field $B_{\max } / B_{0} \gtrsim 1 \%$.

The actual wave magnetic field has been measured directly with a small calibrated receiving loop antenna. The calibration was performed in air using the known simple current distribution near the center of a halfwave electric dipole matched to a signal generator of known output voltage. The calibration is not altered by the presence of the plasma with permeabllity $\mu \simeq \mu_{0}$. The wave magnetic field measurements show consistent but in general smaller values $(\sim 50 \%)$ than the above estimates, even when circular wave polarization and antenna mismatch are taken into account. The discrepancy may lie in the neglect of the electric field energy of the actually oblique whistler modes. Absolute electric field measurements are rather uncertain since a dipole calibration in air is altered by the anisotropic plasma susceptibility and have therefore not yet been performed.

3.3 INTERPRETATION. - The observed self-focusing process is believed to be the result of the reinforcing interaction between the wave induced density depression and the density induced wave refraction. It is theoretically and experimentally known that a density trough can duct whistler waves leading to locally enhanced wave intensities. If the trough is formed by the radiation pressure of the wave the interaction is unstable.

For the present experiment the wave intensity is nonuniform from the beginning having a maximum at the exciter antenna. At large amplitudes the electron motion exhibits an oscillation around a guiding center which drifts away from the region of high field intensities [20]. The drift velocity is the result of second-order terms on the momentum equation $\left(\mathbf{v}_{1} \cdot \nabla \mathbf{v}_{1}, \mathbf{v}_{1} \times \mathbf{B}_{1}\right)$ that become important when the wave energy density $B_{1}^{2} / \mu_{0}$ is comparable to the kinetic particle energy density $n_{\mathrm{e}} k T_{\mathrm{e}}$. This situation occurs initially near the antenna. The wave pressure $\nabla_{\|}\left(B_{1}^{2} / \mu_{0}\right)$ expels electrons from the vicinity of the antenna. The drifting electrons confined by the static magnetic field (electron cyclotron radius $r_{\mathrm{ce}} \simeq 0.2 \mathrm{~mm}$ ) cause a space charge field which accelerates ions thereby giving rise to a density depression which starts the duct. The wave refracts into the density depression and enhances the pressure at the duct front. The process convects, i. e. wave pressure pushes electrons out along $\mathbf{B}_{0}$ and the resulting density depression guides the wave energy flux to the duct front which eventually reaches the end of the plasma device.

The axial propagation velocity of the duct front $\left[v_{\mathrm{d}} \simeq 100 \mathrm{~cm} / 40 \mu \mathrm{s} \simeq 2.5 \times 10^{6} \mathrm{~cm} / \mathrm{s}\right.$ in figure 6$]$ is, 
because of the ion inertia, much smaller than the electron thermal velocity. However, the duct velocity is also much larger than sound speed,

$$
\left[k\left(T_{\mathrm{e}}+T_{\mathrm{i}}\right) / m_{\mathrm{i}}\right]^{1 / 2} \simeq 7.8 \times 10^{4} \mathrm{~cm} / \mathrm{s}
$$

Since the ion cyclotron radius $\left(r_{\mathrm{ci}} \simeq 6 \mathrm{~cm}\right)$ is larger than the duct width ions can leave the duct across $\mathbf{B}_{0}$. Charge neutrality must be preserved by enhanced electron cross-field diffusion. The radial ion motion is consistent with the fast duct formation and the small particle losses observed after the recovery of the density profile.

The observed electron heating strongly correlates with the duct formation and may, therefore, not be simple collisional heating. The temperature rise also contributes to the formation of the density depression since at equilibrium $p=n_{\mathrm{e}} k T_{\mathrm{e}}=$ const. In highly collisional plasmas where the mean free path is much smaller than the gradient scale length of the rf intensity thermal effects alone can lead to filamentation [21-23]. However, this is not the case in the present weakly collisional plasma (mean free path $\sim 20 \mathrm{~cm}$ ) which, for example, becomes evident from the unrelated axial density and temperature profiles and the fast time scale of duct formation compared with equilibration. Collisional effects probably cause the slow broadening of the duct in time.

The wave pressure nonlinearity arises from time averaging quadratic terms in the applied frequency, $\left\langle\cos ^{2} \omega t\right\rangle$. Second harmonic generation is a process of the same order of nonlinearity. The distortions in the interferometer traces (Fig. 6) are direct evidence of this nonlinear process. For $\omega / \omega_{\mathrm{c}}<0.5$ the second harmonic is a propagating mode which is also confined to the duct.

4. Cherenkov instability of oblique whistlers. The present model for the generation of auroral hiss is that of a collective interaction between a stream of precipitating electrons and whistler waves in the ambient background plasma [10]. A Cherenkov-type electrostatic instability arises when the electron beam velocity matches the parallel phase velocity of an oblique whistler whose velocity is small for propagation angles near the resonance cone $\left(\cos \theta \simeq \omega / \omega_{\mathrm{c}}\right)$. Many observations from ground, rockets and satellites are consistent with the above model $[4,15,24]$. The measurements usually consist of noise frequency spectra and electron pitch angle distribution functions. There are fundamental limitations of measuring wave normals or rays from a single observation point. These essential aspects of the instability are usually inferred so that a conclusive proof of the mechanism cannot be given. It will be shown that a carefully designed laboratory experiment can supply this and other complementary information and thereby contribute to the understanding of the VLF hiss phenomenon.
In the present experiment an energetic electron beam is injected into a cold dense, nearly collisionless magnetoplasma. Beam and plasma dimensions are so large that oblique whistler wave propagation and growth are readily observable. In this aspect the present experiment fundamentally differs from most previous laboratory experiments with small diameter beam-plasma systems which are restricted to onedimensional wave propagation [25-27]. The present situation models in many aspects the conditions inside an auroral arc. It does not apply to the wave propagation and refraction effects outside the arc since beam and plasma diameters are identical.

First, the dynamics of the beam and background plasma are investigated. It is concluded that the simultaneously occuring short-wavelength electrostatic instability near the electron plasma frequency $\left(\omega_{p}>\omega_{c}\right)$ is mainly responsible for the scattering of the beam and the saturation of the oblique whistler instability.

Then the observations on the beam-generated broadband of noise are presented. By analyzing the frequency and wavevector spectrum, i. e. the dispersion $\omega(\mathbf{k})$, the noise below $\omega=\omega_{\mathrm{c}}$ is identified as whistler waves propagating near the oblique resonance cone with parallel phase velocity slightly less than beam velocity. This is a direct confirmation of the essential prediction from the instability theory.

Finally, wave propagation experiments are performed by launching coherent test waves from a small antenna in the unstable region. These measurements yield the growth rate. The connection and relevance of the laboratory results on observations in space are discussed in the last section.

4.1 Beam and Plasma Properties. - The beamplasma system is produced repetitively by a double pulse technique. The first voltage pulse applied to the cathode generates a background afterglow plasma which is cold $\left(k T_{\mathrm{e}} \simeq k T_{\mathrm{i}} \simeq 1 / 4 \mathrm{eV}\right)$, dense

$$
\left(10^{9}<n_{\mathrm{e}}<2 \times 10^{10} \mathrm{~cm}^{-3}, 1<\omega_{\mathrm{p}} / \omega_{\mathrm{c}}<6\right),
$$

weakly collisional (electron-neutral collisions

$$
v_{\mathrm{en}} / \omega_{\mathrm{c}} \simeq 10^{-4}
$$

Coulomb collisions $v_{\mathrm{ei}} / \omega_{\mathrm{c}} \simeq 10^{-3}$ ) and free of low or high frequency instabilities. At a desired afterglow time a second voltage pulse is applied to the cathode whereby a beam of fast electrons is injected along $\mathbf{B}_{0}$ into the afterglow plasma. Beam and background plasma parameters are determined from plane Langmuir probes which can be positioned radially and axially. Independently, the density is determined from dispersion measurements of whistler waves, Langmuir waves and Bernstein waves, and from resonance cone measurements. The basic parameters are summarized in table I.

As soon as the beam is injected it strongly interacts with the background plasma and both beam and plasma properties change from their initial properties 
TABLE I

Basic parameters of beam and background plasma.

Gas, pressure

Background plasma density

Electron temperature

Magnetic Field

Ratio of plasma to cyclotron frequency

Electron-neutral collision frequency

(i) Absolute

(ii) Normalized

Electron mean free path

Electron beam energy

Normalized beam density

Beam temperature

Beam electron mean free path for

(i) elastic collisions

(ii) ionizing collisions

Krypton, $2.5 \times 10^{-4}$ torr

$n_{\mathrm{e}}=10^{9}$ to $2 \times 10^{10} \mathrm{~cm}^{-3}$

$k T_{\mathrm{e}} \simeq 2 \mathrm{eV}$

$B_{0}=75 \mathrm{G}$

$1<\omega_{\mathrm{p}} / \omega_{\mathrm{c}}<6$

$v_{\mathrm{en}} \simeq 4 \times 10^{5} \mathrm{~s}^{-1}$

$v_{\mathrm{en}} / \omega_{\mathrm{c}} \simeq 3 \times 10^{-4}$

$l_{\mathrm{e}} \simeq 200 \mathrm{~cm}$

$e V_{\mathrm{b}}=0$ to $50 \mathrm{eV}$

$n_{\mathrm{b}} / n_{\mathrm{e}} \simeq 1 \%$

$k T_{\mathrm{b}} \simeq 0.5$ to $>8 \mathrm{eV}$

$l_{\mathrm{b}} \simeq 90 \mathrm{~cm}$

$l_{\mathrm{b}}^{\prime} \simeq 300 \mathrm{~cm}$

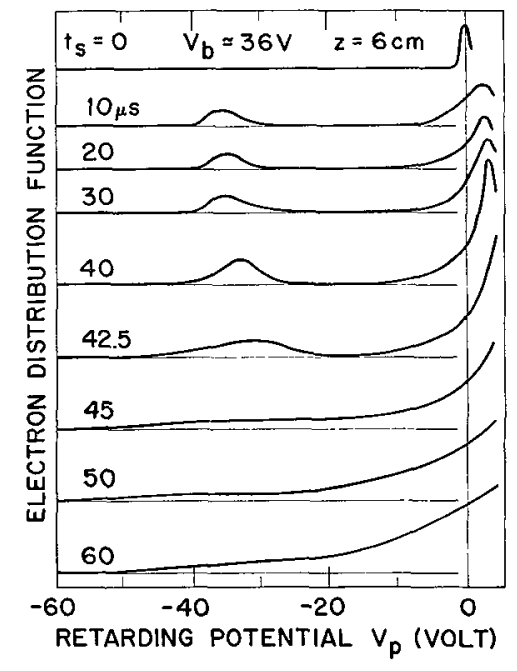

FIG. 11. - Electron distribution function $f_{\mathrm{e}}(V)$, where $V=(m / 2 e) v_{z}^{2}$, sampled at different times $t_{\mathrm{s}}$ after the turn-on of the beam pulse. The figure demonstrates the broadening of the beam and heating of the background plasma in time at a fixed distance $z$ from the anode. $n_{\mathrm{e}} \simeq 6 \times 10^{9} \mathrm{~cm}^{-3}$.

to new steady-state values. Figure 11 shows the electron distribution function sampled at different times $t_{\mathrm{s}}$ after the step-like turn-on of the beam voltage $V_{\mathrm{b}}$ at an afterglow time $t_{\mathrm{a}}=15 \mathrm{~ms}$. The distribution function is obtained by differentiating the I-V characteristics of a one-sided plane Langmuir probe located at $z=7 \mathrm{~cm}$ from the cathode. One can see that initially $\left(0<t_{\mathrm{s}}<30 \mu \mathrm{s}\right)$ a cold electron beam is injected into a cold background plasma

$$
\left(k T_{\mathrm{b}} \simeq k T_{\mathrm{e}} \ll e V_{\mathrm{b}}\right) \text {. }
$$

This system is highly unstable to electrostatic instabilities. With increasing time the beam broadens $\left(t_{\mathrm{s}} \simeq 40 \mu \mathrm{s}\right)$, becomes the tail of a distribution with continuously decreasing slope, $\partial f_{\mathrm{e}} / \partial v_{z}<0$. On the same time scale, the background electrons are strongly heated such that both distributions finally merge. One also observes that beam electrons are accelerated to energies larger than their initial injection energy. This fact as well as the short penetration length of the beam compared with the electron mean free path indicate that the beam is scattered by electrostatic instabilities rather than by collisional processes.

On a longer time scale, however, the beam electrons undergo collisions with the low pressure background gas. When the beam energy exceeds the ionization potential $\left(E_{\mathrm{i}} \simeq 14 \mathrm{eV}\right.$ in krypton), the plasma density increases. Ionization phenomena become noticeable for $t_{\mathrm{s}} \gtrsim 50 \mu \mathrm{s}$. When a $40 \mathrm{eV}$ beam pulse is applied for approx. $500 \mu \mathrm{s}$, which are the typical conditions of the discharge pulse, the density rises to the value at $t_{\mathrm{a}}=0\left(n_{\mathrm{e}} \simeq 2 \times 10^{11} \mathrm{~cm}^{-3}\right)$. For the purpose of the present experiment, however, the initial period $\left(0<t_{\mathrm{s}} \lesssim 50 \mu \mathrm{s}\right)$ of the beam injection is of particular interest since unstable distribution functions are clearly observabie and collective processes dominate. This period is long compared to the time scale of the rf instabilities.

The spatial properties of beam and plasma distributions at a given time $t_{\mathrm{s}}=10 \mu \mathrm{s}$ are shown in figure 12. With increasing distance $z$ from the injection

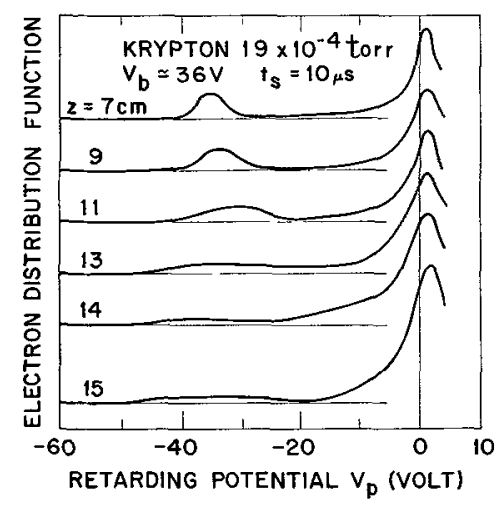

Frg. 12. - Electron distribution function sampled at different distances $z$ from the cathode at a fixed time $t_{\mathrm{s}}$ after beam injection. The figure shows that beam and background heating occur over distances short compared to the electron mean free path $\left(l_{\mathrm{e}} \gtrsim 90 \mathrm{~cm}\right) . n_{\mathrm{e}} \simeq 2 \times 10^{9} \mathrm{~cm}^{-3}$.

point the beam broadens and the background electron temperature and density build up. Unstable beam distributions only persist up to distances $z \approx 20 \mathrm{~cm}$ from the cathode. At the end of the device $(z \simeq 250 \mathrm{~cm})$ the beam has nearly vanished and only a very small tail of energetic electrons is noticeable $\left(n_{\mathrm{b}} / n_{\mathrm{e}} \simeq 10^{-3}\right)$. By rotating the plane probe with respect to $\mathbf{B}_{0}$ it is found that the beam is scattered both in parallel and 
perpendicular velocity space. No back scattered fast electrons are observed.

The strong scattering of the injected beam has further consequences on the background plasma. The injected beam current cannot be returned through the end electrode or the chamber wall. Just like in an unbounded plasma the return current has to be provided by the background plasma. The background electrons drift opposite to the beam electrons toward the gridded anode. The drift velocity is smaller than the beam velocity by the density ratio $\left(n_{\mathrm{b}} / n_{\mathrm{e}} \simeq 10^{-2}\right)$ and lies, in general, between the ion sound and electron thermal velocities. Since the electrons have been heated by the injected beam the return current drives an ion acoustic instability. Strong three-dimensional ion sound turbulence is observed. However, these observations go beyond the scope of the present whistler instability and will be presented elsewhere [28].

4.2 InStability ANALYSIS. - The onset of an instability upon beam injection becomes apparent through the large rf noise level detected with electric or magnetic antennas inside the plasma. Figure $13 a$ shows the coincidence between rf noise and electron beam. The top trace is the rf potential fluctuation which, for purpose of display, has been heterodyned with a local oscillator at $f=100 \mathrm{MHz}\left(\omega / \omega_{\mathrm{c}}=0.47\right)$.

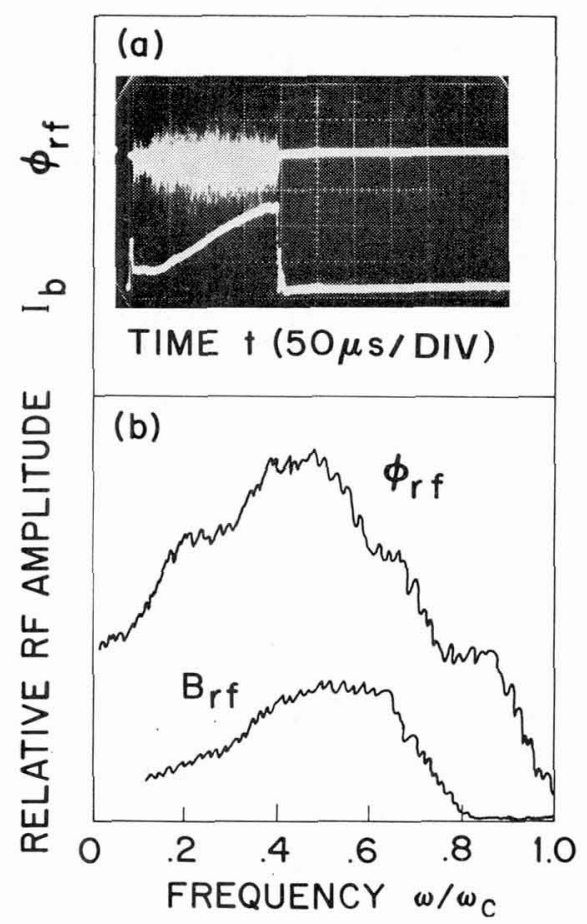

FIG. 13. - (a) Rf noise at $\omega / \omega_{\mathrm{c}}=0.47$ (top trace) and beam current pulse (bottom trace) vs. time, showing the cause-effect relationship between beam and rf emission. $n_{\mathrm{e}} \simeq 6 \times 10^{9} \mathrm{~cm}^{-3}$. (b) Frequency spectrum of the spontaneous emission sampled at $t_{\mathrm{s}}=100 \mu \mathrm{s}$ after beam injection. $\varphi_{\mathrm{rf}}$ is the electrostatic signal detected in the instability region $\left(e\left|\varphi_{\mathrm{rl}}\right| / k T_{\mathrm{e}} \simeq 5 \times 10^{-3}\right)$ and $B_{\mathrm{rP}}$ is the wave magnetic field of whistlers detected $125 \mathrm{~cm}$ away from the excitation region. $\left|\boldsymbol{B}_{\mathrm{r}}\right| / \boldsymbol{B}_{0} \simeq 10^{-5}, \boldsymbol{B}_{0}=75 \mathrm{G}$, $\omega_{\mathrm{p}} / \omega_{\mathrm{c}} \simeq 5$.
The bottom trace shows the beam current pulse applied at $t_{\mathrm{a}}=10 \mathrm{~ms}$ in the afterglow of the main discharge. After an initial transient, the current is constant during the first $50 \mu \mathrm{s}$ and then rises as the density builds up. As outlined earlier, the beam current is balanced by the return current to the anode which is limited by the electron saturation current of the background plasma. The comparison between the two traces clearly shows that the rf is generated only in the presence of the electron beam. This coincidence holds for a wide range of frequencies extending from the regime of ion acoustic waves to that of electron plasma waves.

The high frequency signals are applied to a spectrum analyzer with fast output response $(300 \mathrm{kHz}$ bandwidth). Figure $13 b$ shows the frequency spectrum in the whistler wave regime sampled at $t_{\mathrm{s}}=100 \mu \mathrm{s}$ after turn-on of the beam pulse. The top trace is the rf potential from a short-wire antenna near the region of beam injection, the bottom trace is the magnetic loop signal detected in the middle of the device at $z=125 \mathrm{~cm}$ from the cathode. The broadband character of the instability is apparent. The rf.magnetic field spectrum is cut off beyond $\omega / \omega_{\mathrm{c}} \simeq 0.85$ which can be the result of refraction and damping of whistlers propagating from the beam injection region to the center of the device where the distribution is stable.

Although, in general, the shape of the whistler wave spectrum depends on beam and plasma parameters there is no particular structure noticeable near the characteristic frequencies, the lower hybrid

$$
\left(\omega_{l \mathrm{~h}}^{-2}=\omega_{\mathrm{pi}}^{-2}+\omega_{\mathrm{ce}}^{-1} \omega_{\mathrm{ci}}^{-1} ; \omega_{l \mathrm{~h}} / 2 \pi \simeq 1 \mathrm{MHz}\right)
$$

and the electron cyclotron frequency

$$
\left(\omega_{\mathrm{ce}} / 2 \pi=210 \mathrm{MHz}\right) \text {. }
$$

The noise spectrum extends beyond the cyclotron frequency up to the electron plasma frequency with potential fluctuations comparable to those in the whistler branch.

In order to identify the rf noise signals as waves, the spatial properties have been investigated with correlation techniques. Two identical movable antennas are used whose noise signals are passed through narrowband amplifiers, multiplied, time averaged on the high frequency time scale and then sampled and averaged on the longer time scale of the beam pulse. A typical cross spectral function is plotted vs. axial position in figure $14 a$. One can see that the noise correlates for a few wavelengths along the beam direction. By measuring the wavelengths of different frequency components, the dispersion $\omega\left(k_{\|}\right)$is found. Figure $14 b$ shows that the parallel phase velocity is slightly below but close to the beam velocity. By inserting a delay line in one channel it is also verified that waves and beam travel in the same direction. These properties are characteristic for a Cherenkov type beam-plasma instability.

So far, one component, $k_{\|}$, of the wave vector has been determined. The other component, $k_{\perp}$, is found 

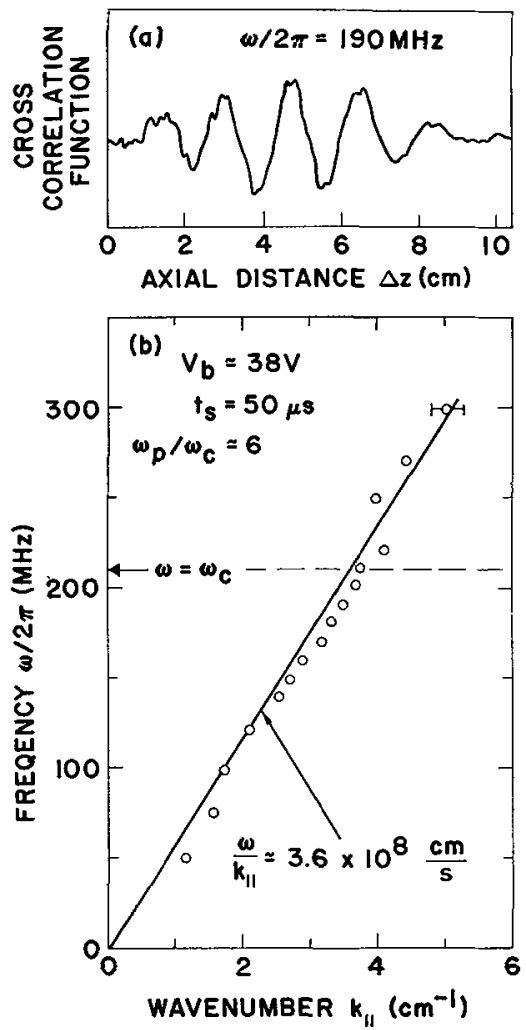

Fig. 14. - (a) Cross spectral function between two narrowband noise signals obtained from a fixed reference antenna and an identical antenna movable along $\mathbf{B}_{0}$. The parallel wavelength $\left(\lambda_{\|}=1.75 \mathrm{~cm}\right)$ is much shorter than that of the whistler propagating along $\mathbf{B}_{0}\left(\lambda_{\mathrm{w}} \simeq 7.9 \mathrm{~cm}\right), \omega / \omega_{\mathrm{c}}=0.9, \omega_{\mathrm{p}} / \omega_{\mathrm{c}} \simeq 6$, $z \simeq 10 \mathrm{~cm},(b)$ Dispersion $\omega$ vs. $k_{\|}$of the spontaneous noise emission. The parallel phase velocity $\omega / k_{\|}$is close to, but below the beam velocity.

by performing two-dimensional cross correlation measurements. Figure $15 a$ shows a map of the location of minima and maxima of the cross spectral function in the $r-z$ plane. One finds that the phase fronts are inclined with respect to the magnetic field. The wavenormal or $\mathbf{k}$ makes an angle $\theta$ with respect to $\mathbf{B}_{0}$ which is close to but below the angle for oblique resonance, $\theta=\arccos \left(\omega / \omega_{\mathrm{c}}\right)$. According to cold plasma theory [4] the dispersion for oblique whistlers is given by

$$
\frac{k^{2} c^{2}}{\omega^{2}}=1+\frac{\omega_{\mathrm{p}}^{2}}{\omega\left(\omega_{\mathrm{c}} \cos \theta-\omega\right)} .
$$

With the experimental parameters $\omega, \omega_{\mathrm{p}}, \omega_{\mathrm{c}}$ given in figure 15 and the measured oblique wavelength $\lambda \simeq 2.2 \mathrm{~cm}$ the calculated angle $\theta$ is found to be $\theta=38.9^{\circ}$ which is in good agreement with the measured value $\theta=38.7^{\circ}$. The angle for oblique resonance is $\theta=40.5^{\circ}$.

By measuring various phase front maps at different frequencies one finds that the propagation angle changes with frequency as shown in figure $15 b$. Over a wide range of frequencies the rf noise is thus identified as whistler waves propagating near the

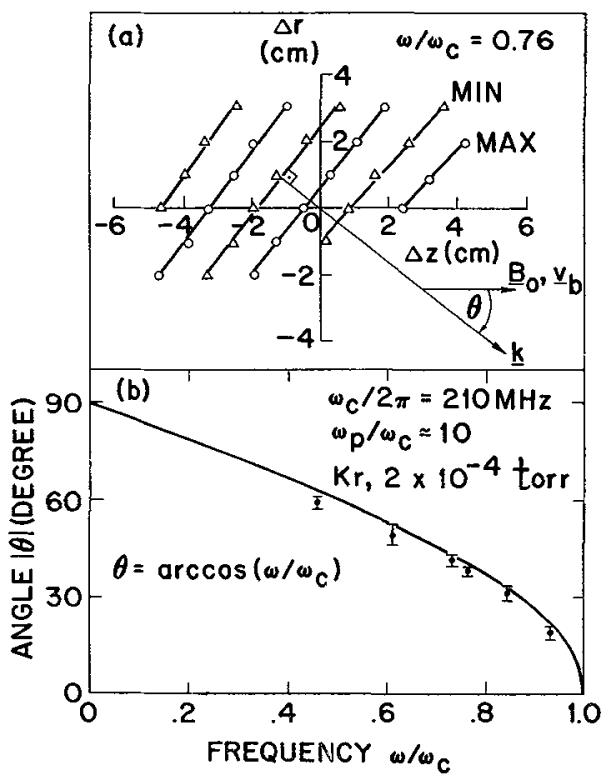

FIG. 15. - Phase fronts of the spontaneously excited waves in the $r-z$ plane obtained from two-dimensional narrowband cross spectral function measurements. The wave normal makes an angle $\theta$ with respect to $\mathbf{B}_{0}$ which is close to the oblique phase velocity resonance cone angle, $\theta=\operatorname{arc} \cos \left(\omega / \omega_{\mathrm{c}}\right)$. (b) Propagation angle $\theta$ at different components $\omega / \omega_{\mathrm{c}}$. The measurement shows that the broadband noise consists of whistlers near the oblique resonance. $V_{\mathrm{b}} \simeq 50 \mathrm{~V}, t_{\mathrm{s}}=30 \mu \mathrm{s}, z \simeq 8 \mathrm{~cm}$.

oblique phase velocity resonance cone. Two further observations are worth pointing out. First, the sign of $\theta$ is found to vary arbitrarily. At some frequencies the waves propagate from the interior of the plasma outward, at other frequencies they propagate inward. Thus, there is no constraint on the direction of wave propagation which is characteristic for an unbounded medium. Second, the amplitude pattern of the cross spectral function shows a systematic frequency dependence. At lower frequencies $\left(\omega / \omega_{\mathrm{c}} \lesssim 0.5\right)$ the correlation length along $\mathbf{B}_{0}$ is larger than across $\mathbf{B}_{0}$, at higher frequencies $\left(\omega / \omega_{\mathbf{c}} \rightarrow 1\right)$ the opposite is observed. This behavior is consistent with the fact that group and phase velocity vectors are essentially perpendicular to one another near the resonance cone. The correlation amplitude is large within the group velocity cone which narrows toward lower frequencies.

A measurement of the absolute value of the instability amplitude involves the knowledge of the coupling properties between antenna and wave. These are difficult to determine theoretically but can be estimated experimentally. For example, as described in the next section, a resonance cone is excited by applying a known small amplitude rf signal to one of the two identical short wire antennas. With the second movable antenna the absolute received signal along the resonance cone is measured. The coupling loss between the two antennas, extrapolated to vanishing probe separation, can be considered as twice the waveantenna coupling factor for a single antenna (typi- 
cally-22 dB). Similar techniques have previously been used for absolute measurements of electron plasma waves [29]. With this calibration procedure the absolute if potential fluctuations are determined to be $\left|\varphi_{\mathrm{rf}}\right| \simeq 8 \mathrm{mV}$ or $e\left|\varphi_{\mathrm{rf}}\right| \mid k T_{\mathrm{e}} \simeq 5 \times 10^{-3}$. At these amplitudes nonlinear wave effects are not considered important.

4.3 Test Wave Propagation. - The propagation of small amplitude phase coherent waves from antennas does not only provide a conclusive test whether the $\mathrm{rf}$ noise is due to incoherent Cherenkov radiation or due to a beam-plasma instability but also provides further information on group and phase velocity directions, growth rates and saturation levels.

When an rf signal $\left(\omega_{l \mathbf{h}}<\omega<\omega_{\mathrm{c}}<\omega_{\mathrm{p}}\right)$ is applied to a small antenna in a Maxwellian plasma, the radiation pattern assumes the shape of two symmetric cones. The origin is at the point source, the axis is long $\mathbf{B}_{0}$ and the half angle is the group velocity resonance cone angle $\theta_{c} \simeq \arcsin \left(\omega / \omega_{c}\right)$. This predicted pattern [13] has been observed both in the near zone [14] and in the far-zone [30]. The field distribution results from the interference of the wide range of oblique eigenmodes $k_{(\theta)}$ excited by an oscillating point source.

In the beam-plasma system the radiation pattern shown in figure 16 is found to be asymmetric along $\mathbf{B}_{0}$. In the direction against the beam a resonance cone is excited just as in the absence of the beam. Figure $16 a$ shows a single localized field maximum when the receiver probe is scanned axially at a fixed radial distance from the cone axis. A two-dimensional scan in the $r-z$ plane (Fig. 16b) shows that the field maxima diverge along two straight lines which, due to azimuthal symmetry represent a cut through a conical radiation pattern. The observed cone angle $\theta_{\mathrm{c}} \simeq 45^{\circ}$ agrees with the expected value $\theta_{\mathrm{c}}=\arcsin$ $\left(\omega / \omega_{c}\right)=45.2^{\circ}$. Since the wave energy flows along the cone through an increasing cross section, the wave amplitude rapidly decreases with distance from the exciter. Contour maps of equal relative if potential $\varphi_{\mathrm{rf}}(r, z) \mid$ are shown in figure $16 e$.

In the direction with the beam the interferometer trace, figure $16 b$, reveals a well defined wave. Its amplitude grows with increasing distance from the exciter, its parallel phase velocity

$$
\left(f \lambda_{\|} \simeq 3.7 \times 10^{8} \mathrm{~cm} / \mathrm{s}\right)
$$

is close to the beam velocity. The phase fronts shown in figure $16 d$ are oblique with respect to $\mathbf{B}_{0}$ and the wave normal makes an angle $\theta=90-\theta_{\mathfrak{c}} \simeq \pm 45^{\circ}$ with respect to $\mathbf{B}_{0}$. Thus, just as in the case of the spontaneous noise emission, the test wave is an oblique whistler wave propagating near the resonance cone. The amplitude distribution shown in figure $16 f$ indicates that the direction of maximum growth, i. e. the group velocity direction is also oblique to $\mathbf{B}_{0}$ and is : nearly perpendicular to the phase velocity

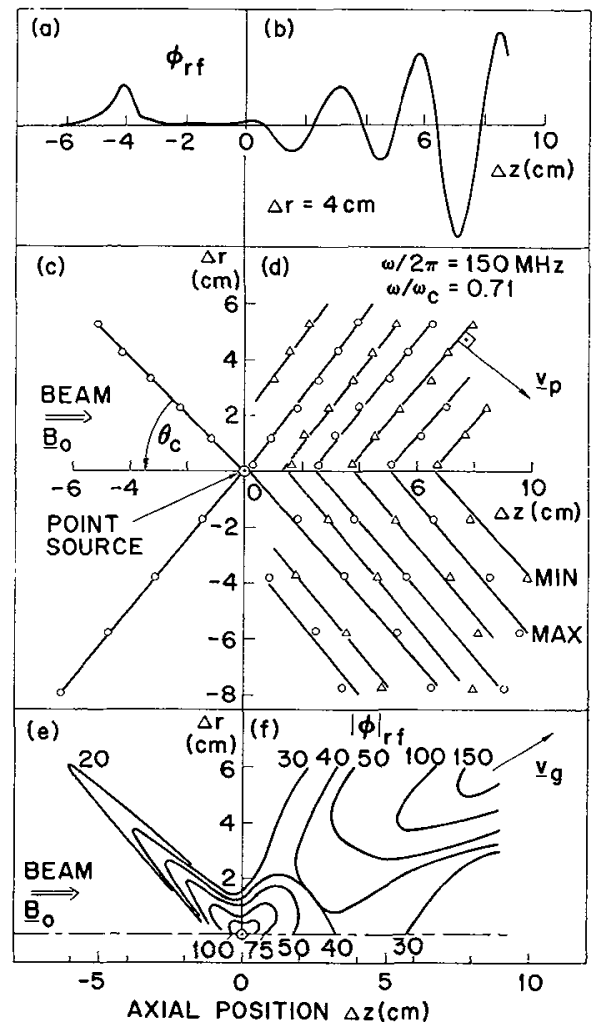

Frg. 16. - Properties of small amplitude test waves excited from a point source in a beam-plasma system. An axial interferometer trace $(a, b)$ shows a highly localized field for propagation against the beam but growing waves for propagation with the beam. A two-dimensional phase front map shows a resonance cone pattern against the beam $(c)$ and oblique waves with the beam $(d)$. A contour map of constant rf amplitudes (arbitrary units) shows amplitude decay along the diverging cone opposing the beam (e) but oblique amplitude growth the beam $(f)$. Note that for the unstable mode $\omega / k_{\|} \lesssim v_{\mathrm{b}}$ and $\mathbf{v}_{\mathrm{g}} \sim \perp \mathbf{v}_{\mathrm{p}}$. Parameters : $\omega_{\mathrm{p}} / \omega_{\mathrm{c}} \simeq 3, t_{\mathrm{s}}=10 \mu \mathrm{s}, t_{\mathrm{a}}=20 \mathrm{~ms}$, $V_{\mathrm{b}} \simeq 38 \mathrm{~V}$.

direction. These observations directly confirm the predicted oblique whistler instability in a beam-plasma system.

The test waves are propagated in the presence of the background noise. Since phase coherent detection is employed and test wave amplitudes above the noise level are chosen, the excited waves are readily distinguished from the background. The fact that the test wave grows indicates that the instability is not yet saturated which is consistent with the relatively small noise amplitudes measured previously. Although the instability shows a broad spectrum of incoherent waves, it cannot be considered as a turbulent system in the sense that strong wave-wave or wave-particle interactions would take place. For the test wave, figure $16 f$ gives an oblique $e$-folding growth length $k_{\mathrm{i}}^{-1} \simeq 3.5 \mathrm{~cm}$ and figure $16 d$ gives the oblique wavelength $\lambda=2 \pi / k_{\mathrm{r}} \simeq 1.8 \mathrm{~cm}$. Because of the conical field distribution the measured normalized growth rate, $k_{\mathrm{i}} / k_{\mathrm{r}} \simeq 8 \times 10^{-2}$, is smaller than that for a plane wave so that a comparison with theory cannot 
be easily given. Nevertheless, one can estimate that a whistler at $\theta=\theta_{\mathrm{c}}=45^{\circ}$ grows by at least $8 e$-folding lengths $(\sim 70 \mathrm{~dB})$ after propagating obliquely through half the column of $20 \mathrm{~cm}$ radius. Further convective growth is limited by the fact that the higher frequency instabilities $\left(\omega \simeq \omega_{\mathrm{p}}\right)$ with short wavelengths, i. e. long interaction lengths modify the beam by quasilinear effects. The growth rate of the hydrodynamic beam-plasma instability is given by [11]

$$
\gamma=\frac{\sqrt{3}}{2^{4 / 3}} \omega_{\mathrm{p}}\left(\frac{n_{\mathrm{b}}}{n_{\mathrm{e}}}\right)^{1 / 3} .
$$

Spatial and temporal growth rates are related by $k_{\mathrm{i}}=\gamma / v_{\mathrm{g}}$ where the group velocity is approximately given by the beam velocity. For $n_{\mathrm{b}} / n_{\mathrm{e}} \simeq 10^{-2}$ one finds $k_{\mathrm{i}}^{-1} \simeq 1 \mathrm{~cm}$ which is much shorter than the whistler wave growth length component along $\mathbf{B}_{0}$.

4.4 Discussion. - The laboratory observations show many similarities with phenomena observed on natural VLF hiss in space. The basic agreement is that VLF hiss is associated with the presence of an electron beam passing through a background plasma. A wide range of beam velocities gives rise to the instability. A broad band of frequencies is excited which shows little structure when the observer is inside the beam plasma system. The frequency spectra observed by James [31] on Isis-1, which passed through regions of electron precipitation, are very similar to the spectra measured in the laboratory. The various characteristic shapes of the frequency-time plots such as saucers and inverted v's shown by Gurnett and Frank [15] are due to propagation effects between the instability region and the moving observer outside the arc. Likewise, observations from ground are strongly affected by wave reflection and refraction processes. The laboratory experiment cannot model such propagation effects involving magnetic field and density gradients and long propagation distances. It does, however, provide much more inside into the generation mechanism of VLF hiss than spacecraft or ground observations. The theoretically predicted coherent Cherenkov-type instability of oblique whistlers is clearly identified through direct measurements of wavenumber spectra, group and phase velocities, growth rates, instability amplitudes and electron distributions. The observations also show that the Cherenkov type instability is dominant over other beam-plasma instabilities, for example the cyclotrontype instability [11] associated with the anomalous Doppler effect, $\omega=k_{\|} v_{\mathrm{b}}-\omega_{\mathrm{c}}$. No evidence is found for this instability, presumably because of its smaller growth rate.

The present experiment also provides insight into the evolution of the beam-plasma system. The electrostatic instabilities of a cold beam plasma system are so strong that the beam rapidly spreads in velocity space. Most observations [32, 33] of the electron pitch-angle distribution in auroral arcs show beams with significant energy spread. Although the mechanism for beam generation is not fully understood, it is possible that the energy spread is the result of electrostatic instabilities as observed in the laboratory.

The finite spatial extent of the beam limits the convective amplitude growth of the oblique whistlers both in the laboratory and in space. However, wave propagation near the resonance cone is slow and the wave amplitude can grow well above the level of incoherent Cherenkov radiation. For very low energy electron beams when the wavelength is within the resonance cone width, the instability in the laboratory vanishes. In spaces, Gurnett and Frank [15] also observe a threshold electron energy level below which VLF hiss is not observed.

5. Summary and conclusions. - The development of a large laboratory plasma provided the basis for studying wave phenomena free from boundary effects and inherent nonuniformities. In the present case obliquely propagating whistler waves have been investigated. It has been shown that these waves are subject to linear instabilities driven by electron beams and to nonlinear instabilities, i. e. self-focusing. The observations should be of importance to whistler wave phenomena in space, both to natural events like VLF hiss and to man-made whistlers launched from spacecraft antennas or by injection of powerful electron beams. However, the final objective of the experiments lies not in the proper scaling of ionospheric parameters but in the fundamental understanding and comparison with theoretical models proposed for various physical processes. In this sense the results are relevant to many situations in plasma physics, e. $g$. rf heating of fusion plasmas and electron beam injection experiments.

Acknowledgments. - The author wishes to thank Dr. R. W. Fredricks for his support and interest in this work. Many fruitful discussions with Dr. C. F. Kennel, Dr. F. V. Coroniti and Dr. J. Maggs are gratefully acknowledged. Thanks are also due to Mr. W. Daley for his assistance in the operation of the device. 


\section{References}

[1] Lutomirski, R. F. and Sudan, R. N., Phys. Rev. 147 (1966) 156.

[2] Palmadesso, P. and Schmidt, G., Phys. Fluids 14 (1971) 1411 ; ibid 15 (1972) 485.

[3] Davidson, R. C., Hammer, D. A., Haber, I. and Wagner, C. E., Phys. Fluids 15 (1972) 317.

[4] Helliwell, R. A., Whistler and Related Ionospheric Phenomena (Stanford University Press, Stanford, California), 1965.

[5] Akhamov, S. A., Sukhorukov, A. P. and Khokhlov, R. V., Usp. Fiz. Nauk 93 (1967) 19 [Sov. Phys.-Usp 14 (1968) 609].

[6] Litvak, A. G., Zh. Eksp. Teor. Fiz. 57 (1968) 629 [Sov. Phys. JETP 30 (1970) 344].

[7] Taniuti, T. and Washimi, H., Phys. Rev. Lett. 22 (1969) $454 ; 22$ (1969) 752; Washim, H., J. Phys. Soc. Japan 34 (1973) 1373.

[8] Hasegawa, A., Phys. Fluids 15 (1972) 870.

[9] BRINCA, A. L., J. Geophys. Res. 77 (1972) 3495.

[10] MAGgs, J., J. Geophys. Res. 81 (1976) 1707.

[11] Mikhailovski, A. B., Theory of Plasma Instabilities (Consultants Bureau, New York), 1974, vol. 1, p. 219. 223.

[12] Stenzel, R. L., Phys. Rev. Lett. 38 (1977) 394.

[13] Kuehl, H. H., Phys. Fluids 5 (1962) 1095.

[14] Fisher, R. K. and Gould, R. W., Phys. Fluids 14 (1971) 857.

[15] Gurnett, D. A. and Frank, L. A., J. Geophys. Res. 77 (1972) 172.

[16] Stenzel, R. L., Phys. Fluids 19 (1976) 857 ; Phys. Fluids, 19 (1976) 865 ; Radio Science 11 (1976) 1045 ; J. Geophys. Res. 82 (October 1977).
[17] Stenzel, R. L., Rev. Sci. Instrum. 47 (1976) 91.

[18] Smith, R. L., Helliwell, R. A. and Yabroff, I. W., $J$. Geophys. Res. 65 (1960) 815.

[19] ADACHI, S., Radio Science 69D (1965) 493 ; ibid. 1 (1966) 671.

[20] Motz H. and Watson, C. J. H., in Advances in Electronics and Electron Physics, edited by L. Marton (Academic, New York), 1967, Vol. 23, p. 153.

[21] Gurevich, A. V., Zh. Eksp. Teor. Fiz. 48 (1965) 701 [Sov. Phys. JETP 21 (1965) 462].

[22] Eremin, B. G. and LitvaK, A. G., Zh. Eksp. Teor. Fiz. Pis. Red. 13 (1971) 693 [Sov. Phys. JETP Lett. 13 (1971) 430.

[23] Perkins, F. W. and Valeo, E. J., Phys. Rev. Lett. 32 (1974) 1234.

[24] LaAspere, T., Johnson, W. C. and Semprebon, L. C., J. Geophys. Res. 76 (1971) 4477.

[25] Malmberg, J. H. and Wharton, C. B., Phys. Fluids 12 (1969) 2600.

[26] Gentle, K. W. and Lohr, J., Phys. Fluids 16 (1973) 1464.

[27] Bollinger, L. D., Carr, W., Liu, H. and SeidL, M., Phys. Fluids 17 (1974) 2142.

[28] Stenzel, R., Phys. Fluids 21 (January 1978).

[29] Malmberg, J. H. and Wharton, C. B., Phys. Rev. Lett. 19 (1967) 775.

[30] Boswell, R. W. and Gonfalone, A., Phys. Lett. 51A (1975) 485.

[31] James, H. G., J. Geophys. Res. 78 (1973) 4578.

[32] Reasoner, D. L. and Chappele, C, R., J. Geophys. Res. 78 (1973) 2176.

[33] Frank, L. A. and ACKerson, K. L., J. Geophys. Res. 76 (1971) 3612 